3. Su J, Thomas F, Kaso W, et al. Thalamus optimized multi atlas segmentation (THOMAS): fast, fully automated segmentation of thalamic nuclei from structural MRI. Neuroimage 2019;194:272-282.

4. Calamante F, Smith R, Liang X, Zalesky A, Connelly A. Track-weighted dynamic functional connectivity (TW-dFC): a new method to study time-resolved functional connectivity. Brain Struct Funct 2017;222:3761-3774.

\section{PROGRESSION OF CLINICAL FEATURES IN LEWY BODY DEMENTIA CAN BE DETECTED OVER SIX MONTHS}

\begin{abstract}
1,2Elie Matar, ${ }^{3}$ Simon R White, ${ }^{4}$ John P Taylor, ${ }^{5}$ Alan Thomas, ${ }^{4}$ lan G McKeith, ${ }^{6} J o s e p h$ PM Kane, ${ }^{2}$ AJ Surendranathan, ${ }^{1}$ Glenda M Halliday, ${ }^{1}$ Simon JG Lewis, ${ }^{2} J o h n$ T O'Brien. ${ }^{1}$ Brain and Mind Centre, University of Sydney, Camperdown, NSW, Australia; ${ }^{2}$ Department of Psychiatry, University of Cambridge, Cambridge, Cambridgeshire, UK; ${ }^{3}$ MRC Biostatistics Unit, University of Cambridge, Cambridge, UK; ${ }^{4}$ Newcastle Translational and Clinical Research Institute, Newcastle University, Newcastle upon Tyne, UK; ${ }^{5}$ Campus for Ageing and Vitality, Newcastle University, Newcastle, UK; ${ }^{6}$ Centre for Public Health, Queen's University Belfast, Belfast, UK
\end{abstract}

10.1136/bmjno-2021-ANZAN.13

Objective This study aimed to quantify the trajectory and magnitude of change of the key clinical features and corresponding symptom domains of Dementia with Lewy bodies (DLB) and Parkinson's disease dementia (PDD) including global cognition, parkinsonism, recurrent visual hallucinations, cognitive fluctuations and sleep disturbance.

Methods 116 patients with Lewy body dementia (DLB $=72$, $\mathrm{PDD}=44$ ) underwent assessment at baseline, 3 and 6 months as part of a prospective multi-centre randomized control trial. Linear mixed models were constructed for core outcome measures using the Mini-Mental State Examination (MMSE), motor section of the Unified Parkinson's disease rating scale (UPDRS-III), Dementia Cognitive Fluctuations Scale (DCFS) and the Neuropsychiatric Inventory (NPI).

Results Within the timeframe of our study (6 months) we were able to identify a significant cognitive decline of 1.3 points on the MMSE $(\mathrm{P}=0.002)$ and significant worsening of motor parkinsonism with an increase in UPDRS-III score of 3.2 points $(\mathrm{P}=0.018)$. Fluctuation severity also increased using the DCFS with a 6 month change in score of 1.3 points $(\mathrm{P}=0.001)$. Uniquely, a signal for increased severity of sleep symptoms of 1.2 points (NPI-sleep) was also detectable $(\mathrm{P}=0.04)$. Significant changes in neuropsychiatric symptoms were not detected. There was no difference in rates of change of scores between DLB and PDD.

Conclusion Clinically significant rates of change in core clinical features can be detected and quantified in Lewy body dementia over a relatively short period (6 months) using common clinical instruments, and thus may be useful as clinical endpoints for therapeutic trials of disease modifying and symptomatic agents.

\section{VISUALISING THE TOPOGRAPHIC PATTERN OF TAU DEPOSITION IN PATIENTS WITH PROGRESSIVE SUPRANUCLEAR PALSY USING PI2620-PET}

1,2Lucy Vivash, ${ }^{2}$ Huiliang Tang, ${ }^{1,2}$ Kelly Bertram, ${ }^{1,2}$ Terence J O'Brien. 'Monash University, Melbourne, VIC, Australia; ${ }^{2}$ Alfred Hospital, Melbourne, VIC, Australia

\subsection{6/bmino-2021-ANZAN.14}

Objectives The accumulation of tau in the basal ganglia and later cerebellum and frontal cortex is a key pathogenic mechanism in Progressive Supranuclear Palsy (PSP). The ability to detect tau in the living brain has the potential to revolutionise the diagnosis of PSP and other tauopathies, and to monitor the effectiveness of therapeutic interventions targeting tau-based mechanisms. This study investigates the use of a new tau-specific PET radiotracer, PI-2620, as a tool for visualising tau in the living brain.

Methods Ten patients with PSP (age 62-75 years, 6 male) underwent a 60 minute dynamic PET scan with PI2620 $(185 \mathrm{MBq})$. The dynamic PET data was processed in to a single parametric image of binding potential (BP) using the simplified reference tissue model and the corpus callosum as the reference region.

Results Visual inspection of the images showed clear uptake in the basal ganglia nuclei. BP was highest in pallidum (1.99), putamen (1.67), thalamus (1.63), substantia nigra (1.62), cerebellum (1.6) and caudate (1.55), compared to 1.2-1.5 across the cortical regions. Higher $\mathrm{BP}$ in the basal ganglia nuclei were associated with higher scores on the PSPRS (putamen, $\mathrm{r}=0.77, \mathrm{p}=0.01)$.

Conclusion PI2620-PET shows promising potential as a technique for specifically imaging and quantitating the topographic pattern of tau distribution in patients with PSP. Further studies are needed to evaluate its use as a diagnostic and treatment monitoring tool for PSP and other tauopathies.

\section{GUT MICROBIOTA AND NUTRITIONAL PROFILES OF PARKINSON'S DISEASE PATIENTS}

${ }^{1,2}$ Michal Lubomski, ${ }^{3}$ Xiangnan $\mathrm{Xu},{ }^{4}$ Andrew J Holmes, ${ }^{3}$ Samuel Mueller, ${ }^{3}$ Jean Yang, ${ }^{1,2}$ Carolyn M Sue, ${ }^{2}$ Ryan L Davis. 'Neurology Department, Royal North Shore Hospital, St Leonards, NSW, Australia; ${ }^{2}$ Department of Neurogenetics, Kolling Institute, University of Sydney and Royal North Shore Hospital, Sydney, NSW, Australia; ${ }^{3}$ School of Mathematics and Statistics. Sydney Precision Bioinformatics, University of Sydney, Camperdown, NSW Australia; ${ }^{4}$ School of Life and Environmental Sciences, The Charles Perkins Centre, University of Sydney, Sydney, NSW, Australia

\subsection{6/bmjno-2021-ANZAN.15}

Objectives Imbalances in the composition of the gut microbiome (GM) from Parkinson's disease (PD) patients have been reported previously. Collectively, the limited literature indicates a reduction in short-chain fatty-acid-producing bacteria that negatively influence colonic permeability and inflammation. We investigated GM composition in association with various clinical features and nutritional data in a large cross-sectional Australian PD cohort, to determine whether short-chain fattyacid-producing bacteria representation in the GM was altered in association with clinical or nutritional differences between PD patients and controls.

Methods Clinical outcome measures derived from PD-validated questionnaires and stool samples were collected from 103 PD patients and 81 spousal healthy controls (HCs). GM composition, determined from $16 \mathrm{~S}$ amplicon sequencing of the V3-V4 region of stool bacterial DNA, was compared between groups and with clinical outcome measures.

Results We identified significant compositional differences in the GM profiles of PD patients compared to HCs, across order, family and genus taxonomic levels. Multiple taxa were associated with a variety of clinical PD characteristics. Predictive models using GM profiles were developed to identify PD and were improved by incorporating nutritional data. 
Conclusions We identified notable differences in microbial diversity and GM composition in PD patients compared to HCs that, along with nutritional data, enabled the development of predictive modelling to identify PD. These findings further support the GM as a potentially useful biomarker of PD pathophysiology.

\section{GENETIC EPIDEMIOLOGY OF MOTOR NEURON DISEASE: ROYAL BRISBANE AND WOMEN'S HOSPITAL COHORT}

Po Sheng Yang, Robert Henderson, Pamela McCombe. Neurology, Royal Brisbane and Women's Hospital, Brisbane, QLD, Australia

\subsection{6/bmjno-2021-ANZAN.16}

Introduction Motor neuron disease (MND) can be sporadic or familial, with approximately $10 \%$ of patients being familial. A number of genetic variants have been found in patients with or without family history. We present the results of genetic testing of a cohort of MND patients diagnosed at Royal Brisbane and Women's Hospital (RBWH).

Methods The criteria for genetic testing for our cohort were consenting MND patients with or without family history. Patients were tested for C9ORF72 repeat expansions and also with a panel of MND genes. The frequency of genetic variants and their clinical features were analysed and compared.

Results There were 47 patients with a genetic variant. The most common variants were in C9ORF72 (27 patients; 57\%), SOD1 (12 patients; 26\%) and TARDBP (4 patients; 9\%). Other genes with variants were TBK1, SPAST, NEK1 and HSPB1 (one patient for each gene). The median age of onset was 50, 49 and 58 years for C9ORF72, SOD1 and TARDBP, respectively. Survival was 813,850 and 1889 days for C9ORF72, SOD1 and TARDBP, respectively. The frequency of bulbar onset was $26 \% ; 8 \%$ and $50 \%$ for C9ORF72, SOD1 and TARDBP, respectively. A family history of MND was present in $63 \%, 83 \%$ and $50 \%$ of C9ORF72, SOD1 and TARDP patients, respectively.

Conclusion The frequencies of genetic variants in our cohort are similar to what is described in European populations. Identification of genetic variants will aid the understanding of the associated phenotypes and identifying patients for possible therapy.

\section{NEUROPHOBIA: A STUDY OF AUSTRALIAN MEDICAL STUDENTS AND JUNIOR DOCTORS}

${ }^{1}$ Alex Yeung, ' 2 eila Karimi, 'Tissa Wijeratne. 'Western Health, Melbourne, VIC, Australia; ${ }^{2}$ La Trobe University, Melbourne, VIC, Australia

\subsection{6/bmino-2021-ANZAN.17}

Objectives Neurological disorders are the leading cause disability in Australia and the world. Combating the perceived difficulty of neurology or 'neurophobia' and improving physician education is a key component in addressing this problem. We aim to conduct the first study to identify whether neurophobia exists in medical students and junior doctors in an Australian population and try to identify factors that may contribute to this in this population.
Method A 24 question online validated survey was distributed via email broadcast to all medical students and junior doctors at a metropolitan tertiary care centre in Australia. Responses were collected over 6 weeks with weekly reminder emails for 4 weeks after the initial invitation email.

Results 114 medical students and junior doctors participated in the study. Participants perceived neurology as the most difficult medical speciality compared to 10 other medical specialties $(p=0.001)$. The top three factors contributing to this perceived difficulty were: a lack of understanding of neuroanatomy, lack of diagnostic certainty and lack of clinical exposure. $65 \%$ of the participants stated that they had too little planned teaching in neurology with only $36 \%$ of the participants having performed a neurology rotation during medical school.

Conclusion The prevalence of neurophobia in this Australian cohort of medical students and junior doctors is consistent with previous findings from around the world. This concerning finding requires further examination into the contributing factors in order to created trials of targeted interventions in order to resolve this.

\section{OPTIMISING SELECTION FOR EPILEPSY SURGERY}

${ }^{1,2}$ Anthony Khoo, ${ }^{1,2}$ Jane de Tisi, 'Shahidul Mannan, ${ }^{3}$ Aidan O'Keeffe, ${ }^{1,2}$ Josemir W Sander, ${ }^{1,2}$ John S Duncan. 'National Hospital for Neurology and Neurosurgery, Queen Square, London, UK; ${ }^{2}$ Department of Clinical and Experimental Epilepsy, UCL Queen Square Institute of Neurology, London, UK; ${ }^{3}$ Department of Statistical Science, UCL, London, UK

\subsection{6/bmjno-2021-ANZAN.18}

Objective To determine reasons for adult patients with drugresistant focal epilepsy who undergo presurgical evaluation not proceeding with surgery and identify factors that influence this decision.

Methods We analyzed demographic, imaging and electroclinical data on 617 consecutive patients brought to the Queen Square presurgical epilepsy MDT between January 2015 and December 2019. Multivariable logistic regression was performed to identify predictors of not proceeding with surgery, using comparative data from a prospectively-followed cohort of individuals who had epilepsy surgery at the same centre over an identical 5-year period.

Results A definitive decision not to proceed with surgery was made in 315 (51\%) cases. Common reasons behind this were an inability to localise the epileptogenic zone $(n=104)$, multifocal epilepsy $(n=74)$ and patients' decisions not to proceed with intracranial EEG $(n=50)$ or surgery $(n=39)$. Learning disability (OR: 2.35; 95\% CI 1.07-5.16), normal MRI (OR: 6.68; 95\% CI 3.71-12.05), extratemporal epilepsy (OR: 2.93; 95\% CI 1.82-4.71) and bilateral seizure onset zones (OR 3.05 ; 95\% CI 1.41-6.61) were independent predictors of not having surgery. Probability of having surgery in those with normal MRI and extratemporal epilepsy was $<10 \%$. Those who did not proceed to surgery resided in more deprived socio-economic areas (median deprivation decile $40-50 \%$ vs 50-60\%, p <0.05).

Conclusions Although underutilized, epilepsy surgery is only appropriate for selected individuals with drug-resistant focal epilepsy. A predictive model based on demographic, imaging and electroclinical data can help determine those unlikely to be suitable for surgery and aid the decision to refer for more extensive or invasive evaluation. 